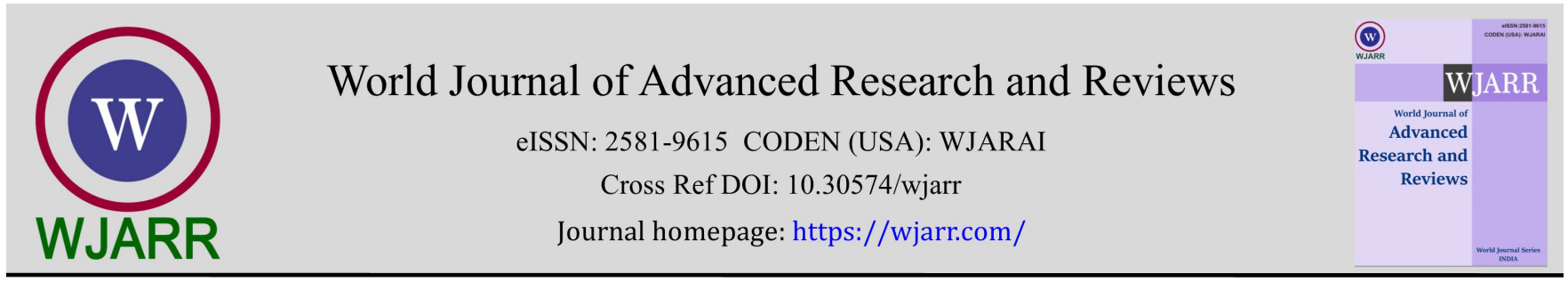

(RESEARCH ARTicle)

\title{
Evaluation of blurring and noise of a medical image using a digital phantom
}

\author{
Cheolpyo Hong * \\ Department of Radiological Science, Daegu Catholic University, Daegu, Republic of Korea.
}

World Journal of Advanced Research and Reviews, 2021, 12(03), 423-426

Publication history: Received on 19 November 2021; revised on 23 December 2021; accepted on 25 December 2021

Article DOI: https://doi.org/10.30574/wjarr.2021.12.3.0735

\begin{abstract}
Blurring and noise are an essential characteristic of a medical image on the imaging system. This study shows the characteristics of blurring and noise of a medical image using a digital phantom. A square-shaped digital phantom was produced with pixels that consist of black and white. The line profile was selected on a binary digital image. An image with noise added was generated and a corresponding line profile was also drawn. The degree of noise was increased using the gaussian noise value. The blurring images obtained by applying gaussian blur to a digital phantom was produced similarities to real images. Also, the degree of blurring was increased using the gaussian blur value. As noise increased, the standard deviation of pixels inside and background the object also increased. However, the boundary of the object was retained. As image blurring increased, the boundary of the object was not clearly distinguished. However, the standard deviation of pixels inside and background the object was retained. When extreme noise and blurring are increased, the resulting images are different. For adding noise, the shape is visually maintained. However, the blurred image does not maintain a square shape. Therefore, it is shown that blurring due to movement of object cannot maintain original form. In the image processing method, the reduction of noise is achieved through blur processing. The noise was reduced through blur processing in the image with noise. The degree of noise decreased, but the ambiguity of the boundary increased.
\end{abstract}

Keywords: Blurring; Noise; Medical image; Digital phantom

\section{Introduction}

Imaging systems of medical area employ the spatial resolution in order to discrimination various small objects [1-3]. The spatial resolution depends on the pixels which are composed of rows and columns. The pixel is the value for the size of image divided by the matrix. The two-dimensional (2D) image is composed of the several pixels [4,5].

The degradation of 2D image is produced by the intrinsic factors of the imaging system. In particular, noise which is unwanted signal from object can be easily observed in medical images [6]. It can be defined as a standard deviation of the object. Such a noise effect can generate the obscurity of a pixel in the object inside and outside. To eliminate the ambiguity of object identification, various noise reduction techniques have been proposed.

Image blurring can also be created due to object motion and imperfections of medical imaging systems [7]. For instance, an object represented by one pixel moves, it is misrepresented by a plurality of pixels. Also, the boundary of the pixel value is also unclear. When image blurring occurs, it becomes difficult to distinguish objects. In particular, the spatial resolution rapidly decreases. However, the comprehensive visualization of characteristics for image blurring and noise are not well described.

In this study, we show the characteristics of blurring and noise of a medical image using a digital phantom.

\footnotetext{
* Corresponding author: Cheolpyo Hong

Department of Radiological Science, Daegu Catholic University, Daegu, Republic of Korea.

Copyright $(2021$ Author(s) retain the copyright of this article. This article is published under the terms of the Creative Commons Attribution Liscense 4.0.
} 


\section{Material and methods}

\subsection{Digital phantom image}

For this study, A square-shaped digital phantom was produced with pixels that consist of black and white, 0 and 255 intensities. The image has a $512 \times 512$ matrix, shown in figure 1(a). The corresponding line profile was selected on a binary digital image, shown in figure $1(\mathrm{~b})$.
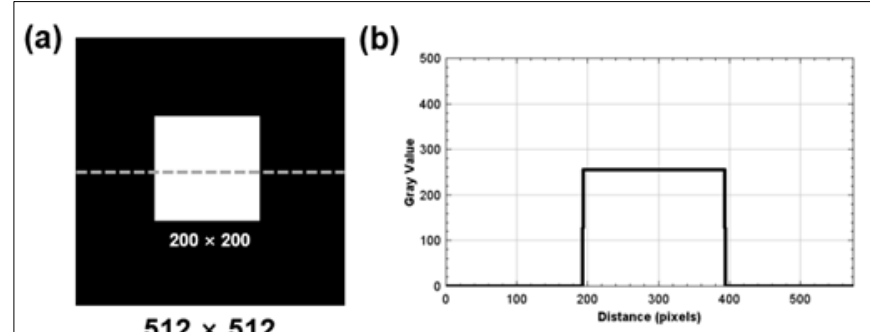

Figure 1 A square-shaped digital phantom and the corresponding line profile

\subsection{The effects of noise}

An image with noise added was generated and a corresponding line profile was also drawn, shown in figure 2 . The degree of noise was increased using the gaussian noise value. A Gaussian noise with a standard deviation of 20,40 , and 60 was applied on the digital image. The gray dotted line shows the line profile.

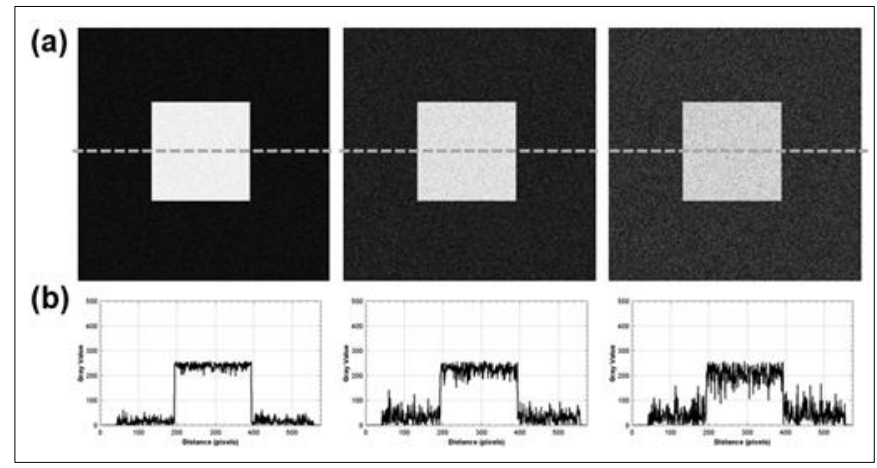

Figure 2 An image with noise added and a corresponding line profile

\subsection{The effects of blurring}

The blurring images obtained by applying gaussian blur to a digital phantom was produced similarities to real images, shown in figure 3 . Also, the degree of blurring was increased using the gaussian blur value. A Gaussian blur with a sigma radius of 4,8 , and 12 was applied on the digital image. The gray dotted line shows the line profile.

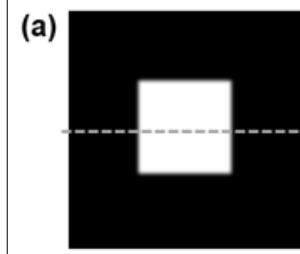

(b)

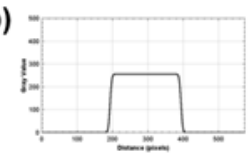

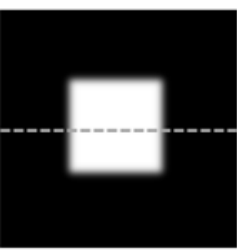

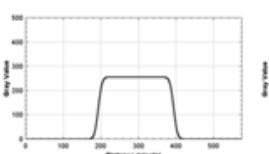

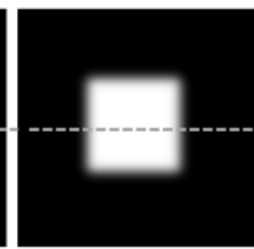

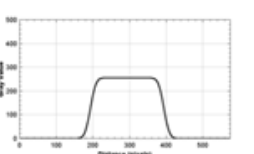

Figure 3 The blurring images and a corresponding line profile 


\section{Results and discussion}

As noise increased, the standard deviation of pixels inside and background the object also increased. However, the boundary of the object was retained. As image blurring increased, the boundary of the object was not clearly distinguished. However, the standard deviation of pixels inside and background the object was retained (Figure 4).

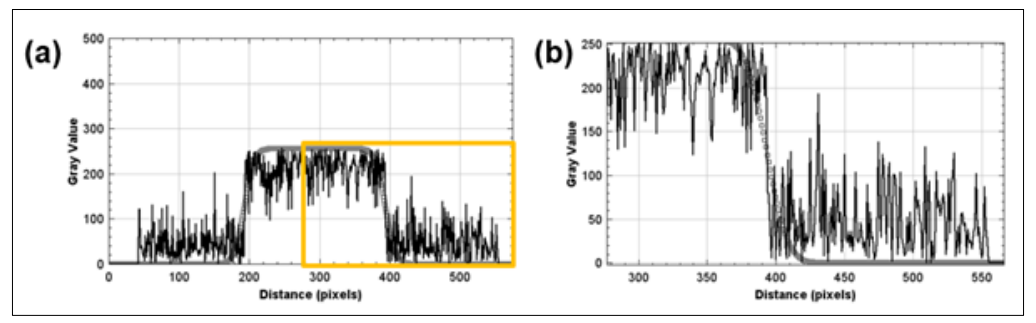

Figure 4 Comparison of noise-added profiles with blurring applied profiles

A Gaussian noise with a standard deviation of 200 and a Gaussian blur with a sigma radius of 50 was applied on the digital image (figure 5). When extreme noise and blurring are increased, the resulting images are different. For adding noise, the shape is visually maintained. However, the blurred image does not maintain a square shape. Therefore, it is shown that blurring due to movement of object cannot maintain original form.

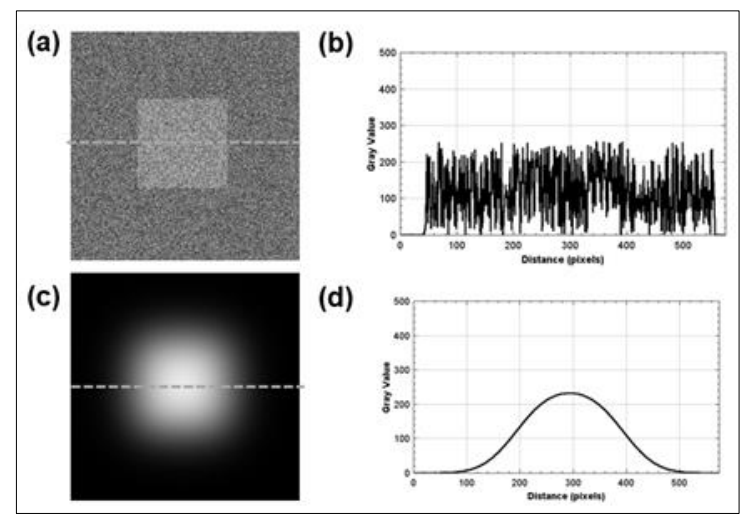

Figure 5 Extremely noise and blurring added image

In the image processing method, the reduction of noise is achieved through blur processing. The noise was reduced through blur processing in the image with noise. The degree of noise decreased, but the ambiguity of the boundary increased (figure 6). The signal size was reduced due to the blur effect applied to the noise, and the background noise was increased. The noise removal resulted in an increase in ambiguity on the boundary and a decrease in signal-tonoise ratio.

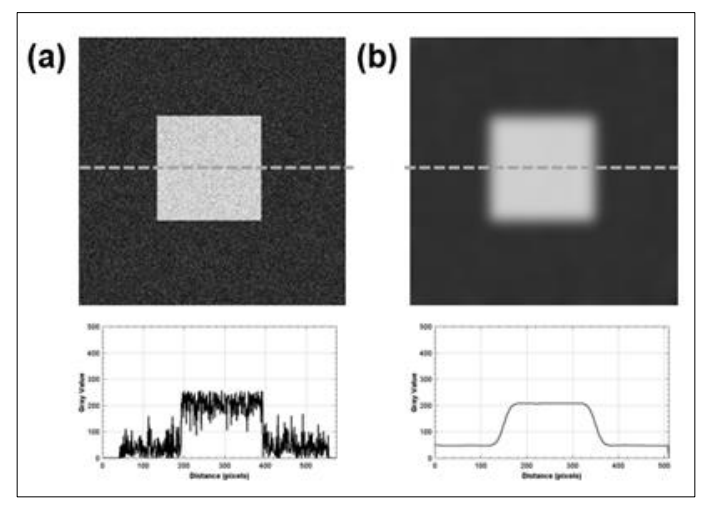

Figure 6 Noise reduction using Gaussian blur 


\section{Conclusion}

This study demonstrates the characteristics of blurring and noise of a medical image using a digital phantom. It may be possible to education for blurring and noise evaluation using digital phantom.

\section{Compliance with ethical standards}

\section{Disclosure of conflict of interest}

None.

\section{Statement of ethical approval}

The present research work does not contain any studies performed on animals/humans subjects by any of the authors.

\section{References}

[1] Bankman I. Handbook of medical image processing and analysis: Elsevier. 2008.

[2] Chow LS, Paramesran R. Review of medical image quality assessment. Biomedical signal processing and control. 2016; 27: 145-54.

[3] Dhawan AP. Medical image analysis: John Wiley \& Sons. 2011.

[4] McAuliffe MJ, Lalonde FM, McGarry D, Gandler W, Csaky K, Trus BL, editors. Medical image processing, analysis and visualization in clinical research. Proceedings 14th IEEE Symposium on Computer-Based Medical Systems CBMS. 2001.

[5] Penney GP, Weese J, Little JA, Desmedt P, Hill DL. A comparison of similarity measures for use in 2-D-3-D medical image registration. IEEE transactions on medical imaging. 1998; 17(4): 586-95.

[6] Sanches JM, Nascimento JC, Marques JS. Medical image noise reduction using the Sylvester-Lyapunov equation. IEEE transactions on image processing. 2008; 17(9): 1522-39.

[7] Semmlow JL. Biosignal and medical image processing: CRC press. 2008. 